\title{
NATURALLY ORDERED BANDS
}

\author{
by J. M. HOWIE
}

(Received 12 April, 1966)

In the terminology of Clifford and Preston [2], a band $B$ is a semigroup in which every element is idempotent. On such a semigroup there is a natural (partial) order relation defined by the rule

$$
e \leqq f \text { if and only if } e f=f e=e .
$$

If the order relation $\leqq$ is compatible with the multiplication in $B$, in the sense that $e \leqq f$ and $g \leqq h$ together imply that $e g \leqq f h$, we shall say that $B$ is a naturally ordered band. The object of this note is to describe the structure of naturally ordered bands.

It is clear that a semilattice is a naturally ordered band. It is also the case that a rectangular band, which it is convenient to define here as a band in which the relation $x y z=x z$ holds identically, is naturally ordered, since in such a band $e \leqq f$ if and only if $e=f$. The structure of a rectangular band can be described completely in terms of sets: it is the cartesian product $I \times J$ of two sets $I$ and $J$, with multiplication defined by

$$
\left(i_{1}, j_{1}\right)\left(i_{2}, j_{2}\right)=\left(i_{1}, j_{2}\right) \text {. }
$$

It is known (Clifford [1], McLean [2]) that an arbitrary band is a semilattice of rectangular bands. Investigations into what Clifford and Preston [2, p. 28] have called the "fine structure" of unions of groups (of which bands are a special case) have been made by Clifford, particularly in the final section of his paper [1], and, more recently, by Fantham [3] and Petrich [7]. Both Fantham and Petrich give descriptions of the structure of certain types of unions of groups in terms of bands, so that their theorems become trivial when applied to bands. Clifford considers the structure of a semigroup which is the disjoint union of an arbitrary semigroup $S_{\alpha}$ and a completely simple semigroup $S_{\beta}$, in which $S_{\alpha} S_{\beta}$ and $S_{\beta} S_{\alpha}$ are both contained in $S_{\beta}$. Some of the steps in the proofs below can be deduced from results of Clifford, but it seemed easier to derive them independently.

The first theorem characterises naturally ordered bands in such a way as to show that they form a subvariety of the variety of bands.

THEOREM 1. $A$ band $B$ is naturally ordered if and only if the identical relation

$$
x z x y x z t z x z=x y x z t z
$$

holds in $B$.

Proof. For any $x, y, z, t$ in $B$, we have

$$
x y x \leqq x, \quad z t z \leqq z .
$$

If $\leqq$ is compatible, it follows that $x y x z t z \leqq x z$, and hence $x z x y x z t z x z=x y x z t z$ as required.

Conversely, if (1) holds identically in $B$, and if $y \leqq x, t \leqq z$, then $y=x y x, t=z t z$. 
Hence

$$
\begin{aligned}
y t=x y x z t z & =x z x y x z t z x z, \quad \text { by }(1), \\
& =x z y t x z,
\end{aligned}
$$

from which it follows that $y t \leqq x z$. Thus $\leqq$ is compatible.

It follows incidentally that not all bands are naturally ordered: the free band on four generators (see Green and Rees [4]) clearly does not satisfy the identical relation (1).

THEOREM 2. Let $Y=\{\alpha, \beta, \gamma, \ldots\}$ be a semilattice and let $\left\{B_{\alpha}: \alpha \in Y\right\}$ be a family of disjoint rectangular bands, indexed by $Y$. If $\alpha>\beta$ in $Y$, let $\phi_{\alpha, \beta}$ be a homomorphism from $B_{\alpha}$ into $B_{\beta}$, and suppose that if $\alpha>\beta>\gamma$ then

$$
\phi_{\alpha, \gamma}=\phi_{\alpha, \beta} \phi_{\beta, \gamma} \text {. }
$$

Let $\phi_{\alpha, \alpha}$ be the identical automorphism of $B_{\alpha}$. Let $S$ be the union of the rectangular bands $B_{\alpha}$ and define the product of two elements $e_{\alpha}$ and $f_{\beta}$ of $S$ (in $B_{\alpha}$ and $B_{\beta}$ respectively) by

$$
e_{\alpha} f_{\beta}=\left(e_{\alpha} \phi_{\alpha, \gamma}\right)\left(f_{\beta} \phi_{\beta, \gamma}\right) \text {, }
$$

where $\gamma=\alpha \beta$, the product of $\alpha$ and $\beta$ in the semilattice $Y$, and where the right-hand product is evaluated in the rectangular band $B_{\gamma}$.

Then $S$ is a naturally ordered band. Conversely, any naturally ordered band can be constructed in this way.

Proof. First, since it is clear that the transitivity condition (2) also holds under the weaker assumption that $\alpha \geqq \beta \geqq \gamma$, the groupoid $S$ whose construction is described in the statement of the theorem is an example of what Fantham [3] calls a mapping semigroup of an array of semigroups over the semilattice $Y$. Hence, by [3, Proposition 3], $S$ is a semigroup. Clearly $S$ is a band, since every element of $S$ belongs to some $B_{\alpha}$.

If $e_{\alpha} \in B_{\alpha}$ and $f_{\beta} \in B_{\beta}$, then $f_{\beta} \leqq e_{\alpha}$ if and only if $f_{\beta}=e_{\alpha} f_{\beta} e_{\alpha}$. In fact, we can show that

$$
f_{\beta} \leqq e_{\alpha} \text { if and only if } \beta \leqq \alpha \text { and } f_{\beta}=e_{\alpha} \phi_{\alpha, \beta} \text {. }
$$

For if $f_{\beta} \leqq e_{\alpha}$ then the multiplication rule (3) implies that $\alpha \beta \alpha=\beta$, from which we deduce that $\beta \leqq \alpha$. Again by (3), we have that

$$
f_{\beta}=\left(e_{\alpha} \phi_{\alpha, \beta}\right) f_{\beta}\left(e_{\alpha} \phi_{\alpha, \beta}\right)=e_{\alpha} \phi_{\alpha, \beta},
$$

since $B_{\beta}$ is a rectangular band. Conversely, if $\beta \leqq \alpha$ and $f_{\beta}=e_{\alpha} \phi_{\alpha, \beta}$, then

$$
e_{\alpha} f_{\beta} e_{\alpha}=\left(e_{\alpha} \phi_{\alpha, \beta}\right) f_{\beta}\left(e_{\alpha} \phi_{\alpha, \beta}\right)=f_{\beta}^{3}=f_{\beta},
$$

and so $f_{\beta} \leqq e_{\alpha}$.

To show that the band $S$ is naturally ordered, suppose that $f_{\beta} \leqq e_{\alpha}$ and $h_{\delta} \leqq g_{\gamma}$, where $g_{\gamma} \in B_{\gamma}$ and $h_{\delta} \in B_{\delta}$; we must show that $f_{\beta} h_{\delta} \leqq e_{\alpha} g_{\gamma}$. By (4), we have that

$$
\beta \leqq \alpha, \quad \delta \leqq \gamma, \quad f_{\beta}=e_{\alpha} \phi_{\alpha, \beta}, \quad h_{\delta}=g_{\gamma} \phi_{\gamma, \delta} \text {. }
$$

Now

$$
e_{\alpha} g_{\gamma}=\left(e_{\alpha} \phi_{\alpha, \alpha \gamma}\right)\left(g_{\gamma} \phi_{\gamma, \alpha \gamma}\right)=p_{\alpha \gamma},
$$

say, and

$$
f_{\beta} h_{\delta}=\left(f_{\beta} \phi_{\beta, \beta \delta}\right)\left(h_{\delta} \phi_{\delta, \beta \delta}\right)=q_{\beta \delta} \text {. }
$$


The natural order relation in the semilattice $Y$ is compatible with the multiplication in $Y$, and so certainly $\beta \delta \leqq \alpha \gamma$. Also

$$
\begin{gathered}
q_{\beta \delta}=\left(f_{\beta} \phi_{\beta, \beta \delta}\right)\left(h_{\delta} \phi_{\delta, \beta \delta}\right)=\left(e_{\alpha} \phi_{\alpha, \beta} \phi_{\beta, \beta \delta}\right)\left(g_{\gamma} \phi_{\gamma, \delta} \phi_{\delta, \beta \delta}\right)=\left(e_{\alpha} \phi_{\alpha, \alpha \gamma} \phi_{\alpha \gamma, \beta \delta}\right)\left(g_{\gamma} \phi_{\gamma, \alpha \gamma} \phi_{\alpha \gamma, \beta \delta}\right) \\
=\left[\left(e_{\alpha} \phi_{\alpha, \alpha \gamma}\right)\left(g_{\gamma} \phi_{\gamma, \alpha \gamma}\right)\right] \phi_{\alpha \gamma, \beta \delta}=p_{\alpha \gamma} \phi_{\alpha \gamma, \beta \delta},
\end{gathered}
$$

and so $f_{\beta} h_{\delta} \leqq e_{\alpha} g_{\gamma}$ as required.

Conversely, if $S$ is a naturally ordered band, then $S$ is, by virtue of the theorem of Clifford [1] and McLean [5], a semilattice $Y$ of rectangular bands $\left\{B_{\alpha}: \alpha \in Y\right\}$. The rectangular bands $B_{\alpha}$ are the $\mathscr{J}$-classes of $S$, and $B_{\alpha} \leqq B_{\beta}$ in the natural order among the $\mathscr{J}$-classes (see [2, $\S 2.1)]$ if and only if $\alpha \leqq \beta$ in the semilattice $Y$.

LEMMA. Let $\alpha, \beta$ be elements of the semilattice $Y$ such that $\beta \leqq \alpha$, and let $e_{\alpha}$ be an arbitrary element of $B_{\alpha}$. Then there exists one and only one element $f_{\beta}$ of $B_{\beta}$ such that $f_{\beta} \leqq e_{\alpha}$.

Proof. If $b_{\beta}$ is an arbitrary element of $B_{\beta}$, then $e_{\alpha} b_{\beta} e_{\alpha} \in B_{\alpha \beta \alpha}=B_{\beta}$, since $\beta \leqq \alpha$. Also $e_{\alpha} b_{\beta} e_{\alpha} \leqq e_{\alpha}$, since

$$
e_{\alpha} \cdot e_{\alpha} b_{\beta} e_{\alpha}=e_{\alpha} b_{\beta} e_{\alpha} \cdot e_{\alpha}=e_{\alpha} b_{\beta} e_{\alpha} .
$$

Suppose now that $f_{\beta}$ and $g_{\beta}$ are two elements of $B_{\beta}$ such that $f_{\beta} \leqq e_{\alpha}, g_{\beta} \leqq e_{\alpha}$. Then

$$
f_{\beta} e_{\alpha}=e_{\alpha} f_{\beta}=f_{\beta}, \quad g_{\beta} e_{\alpha}=e_{\alpha} g_{\beta}=g_{\beta}
$$

and, since $B_{\beta}$ is a rectangular band,

$$
f_{\beta} g_{\beta} f_{\beta}=f_{\beta}, \quad g_{\beta} f_{\beta} g_{\beta}=g_{\beta} .
$$

Since $\leqq$ is by assumption compatible,

$$
f_{\beta} g_{\beta} \leqq f_{\beta} e_{\alpha}=f_{\beta}, \quad f_{\beta} g_{\beta} f_{\beta} \leqq f_{\beta} g_{\beta} e_{\alpha}=f_{\beta} g_{\beta}
$$

Hence

$$
f_{\beta}=f_{\beta} g_{\beta} f_{\beta} \leqq f_{\beta} g_{\beta} \leqq f_{\beta},
$$

from which we deduce that $f_{\beta} g_{\beta}=f_{\beta}$. But, by a similar argument, $f_{\beta} g_{\beta}=g_{\beta}$, and so $f_{\beta}=g_{\beta}$. This completes the proof of the lemma.

Returning now to the proof of Theorem 2, we can, by virtue of the lemma, define a mapping $\phi_{\alpha, \beta}: B_{\alpha} \rightarrow B_{\beta}$ (if $\beta \leqq \alpha$ ) by taking $e_{\alpha} \phi_{\alpha, \beta}$ to be the unique element $f_{\beta}$ of $B_{\beta}$ such that $f_{\beta} \leqq e_{\alpha}$. The compatibility of the order ensures that $\phi_{\alpha, \beta}$ is a homomorphism, while if $\beta=\alpha$ the mapping is the identical automorphism of $B_{\alpha}$. The condition (2) is a direct result of the transitivity of the order.

To verify (3), first notice that if $\gamma \leqq \alpha$ and $e_{\alpha} \in B_{\alpha}$, then $e_{\alpha} b_{\gamma} e_{\alpha} \leqq e_{\alpha}$ for any $b_{\gamma}$ in $B_{\gamma}$, and so $e_{\alpha} \phi_{\alpha, \gamma}=e_{\alpha} b_{\gamma} e_{\alpha}$. If $f_{\beta} \in B_{\beta}$ and if we take $\gamma$ as $\alpha \beta$, we know that $e_{\alpha} f_{\beta} \in B_{\gamma}$. Hence

$$
e_{\alpha} \phi_{\alpha, \gamma}=e_{\alpha}\left(e_{\alpha} f_{\beta}\right) e_{\alpha}
$$

and similarly $f_{\beta} \phi_{\beta, \gamma}=f_{\beta}\left(e_{\alpha} f_{\beta}\right) f_{\beta}$. Hence

$$
\left(e_{\alpha} \phi_{\alpha, \gamma}\right)\left(f_{\beta} \phi_{\beta, \gamma}\right)=e_{\alpha}\left(e_{\alpha} f_{\beta}\right) e_{\alpha} f_{\beta}\left(e_{\alpha} f_{\beta}\right) f_{\beta}=\left(e_{\alpha} f_{\beta}\right)^{3}=e_{\alpha} f_{\beta}
$$

This completes the proof. 
Remark. As can easily be verified, any homomorphism $\phi$ from a rectangular band $I \times J$ into a rectangular band $K \times L$ determines two mappings $\lambda: I \rightarrow K, \mu: J \rightarrow L$ such that

$$
(i, j) \phi=(i \lambda, j \mu)
$$

for every $(i, j)$ in $I \times J$. Conversely, if $\lambda: I \rightarrow K$ and $\mu: J \rightarrow L$ are arbitrary mappings, then (5) defines a homomorphism $\phi: I \times J \rightarrow K \times L$. These statements can alternatively be deduced from a general theorem due to Munn [6] and quoted by Clifford and Preston [2, Theorem 3.11].

\section{REFERENCES}

1. A. H. Clifford, Semigroups admitting relative inverses, Ann. of Math. 42 (1941), 1037-1049.

2. A. H. Clifford and G. B. Preston, The algebraic theory of semigroups, American Mathematical Society Mathematical Surveys No. 7, Vol. 1 (Providence, R.I., 1961).

3. P. H. H. Fantham, On the classification of a certain type of semigroup, Proc. London Math. Soc. (3) 10 (1960), 409-427.

4. J. A. Green and D. Rees, On semigroups in which $x^{r}=x$, Proc. Cambridge Philos. Soc. 48 (1952), 35-40.

5. D. McLean, Idempotent semigroups, Amer. Math. Monthly 61 (1954), 110-113.

6. W. D. Munn, Semigroups and their algebras, Thesis, Cambridge (1955).

7. Mario Petrich, The structure of a class of semigroups which are unions of groups, Notices Amer. Math. Soc. 12 No. 1, Part 1 (1965), p. 102.

UNIVERSITY OF GLASGOW

GlasGow, W.2. 